\title{
Gluteoplastia de aumento: a importância do ensino na formação atual do residente frente à demanda crescente
}

\author{
Buttocks increase: the importance of teaching in current resident facing increased \\ demand
}

\author{
Diogo Almeida Lima ${ }^{1}$ \\ Daniel Akira Minakami ${ }^{1}$ \\ Lucas Chagas Pereira ${ }^{1}$ \\ Maieve Corralo Grando ${ }^{1}$ \\ Ricardo Souza Cruz ${ }^{1}$ \\ André Ramalho Braga ${ }^{2}$ \\ Enéas Cardoso de Almeida \\ NETO $^{2}$ \\ Rodrigo Araújo Guimarães ${ }^{2}$ \\ Ronaldo CarRaRa DE \\ Almeida $^{2}$ \\ Acrysio Peixoto de Souza \\ $\mathrm{NeTO}^{3}$ \\ Cristiam Machado \\ HAESBAERT $^{3}$ \\ Márcio Grave ${ }^{3}$ \\ Ricardo Morgental \\ ZAMBENEDETTI $^{3}$ \\ Marcelo Moreira Cardoso ${ }^{4}$ \\ José Humberto Cardoso \\ RESENDE 5
}

Trabalho realizado no Serviço de Cirurgia Plástica do Hospital dos Servidores do Estado do Rio de Janeiro, Rio de Janeiro, RJ,

Brasil.

Artigo submetido pelo SGP (Sistema de Gestão de Publicações) da RBCP.

Artigo recebido: 30/1/2011

Artigo aceito: 15/3/2011

\begin{abstract}
RESUMO
Introdução: Frente ao aumento na demanda por gluteoplastia de aumento com implantes nos últimos anos, é importante que haja profissionais treinados e capacitados para realizar tal procedimento e suas associações com lipoaspiração/lipoenxertia, com o intuito final de melhorar o contorno do quadril. Método: Ao ver na residência médica o local de ensino apropriado para tal prática, elaboramos o estudo de 20 casos tratados por médicos residentes em treinamento sob supervisão, a fim de observar a aplicabilidade e exequibilidade do método e seus resultados. Resultados: Alcançamos melhoria do contorno glúteo em todos pacientes, o que foi corroborado pelo nível de satisfação elevado de 100\% delas e pequena taxa de complicações, compatíveis com a literatura. Há certo receio no início do aprendizado, devido ao descolamento intramuscular às cegas, mas guiados por parâmetros técnicos e anatômicos precisos e sob supervisão qualificada, conseguiu-se a realização da cirurgia no plano intramuscular adequado, associando a lipoaspiração e lipoenxertia em alguns casos, o que proporcionou ótima cobertura do implante e resultados naturais no contorno final do quadril. Conclusão: $O$ estudo demonstrou a grande aplicabilidade do ensino da gluteoplastia de aumento com implantes ao residente em formação, visto a necessidade de uma formação adequada, completa e segura, para atender à crescente demanda por implantes glúteos observada no mercado atual.
\end{abstract}

Descritores: Nádegas/cirurgia. Implante de Prótese. Próteses e Implantes. Lipectomia.

\begin{abstract}
Introduction: Facing the increasing demand for buttocks augmentation with implants in recent years, it is important that there are enough trained and qualified to perform such a procedure and their associations with liposuction/ fat grafting with an ultimate aim of improving the contour of the hip. Methods: Seeing in the residency teaching sites suitable for such a practice, we make a study of 20 cases treated by resident physicians in training under supervision in order to observe the applicability and feasibility of the method and its results. Results: Achieved the improvement of the gluteal contour in all patients, which was corroborated by the high level of satisfaction $100 \%$ of them and low rate of complications, consistent with the literature. It is known that there is a certain fear in the beginning of learning, due to detachment in blind, but guided by precise anatomical and technical parameters and under qualified supervision, we had the surgery in the plan adequate intramuscular associated with liposuction and fat grafting in some cases, which provided excellent coverage of the implant and natural results in the final contour of the hip. Conclusion: The study showed the wide applicability of the teaching of buttocks augmentation with implants, to the resident in training, given the need for adequate training to meet the growing demand in the market today.
\end{abstract}

Keywords: Buttocks/surgery. Prosthesis Implantation. Prostheses and Implants. Lipectomy.

1. Médico aspirante a membro especialista da Sociedade Brasileira de Cirurgia Plástica; Residente do $3^{\circ}$ ano de Cirurgia Plástica do Hospital dos Servidores do Estado do Rio de Janeiro, Rio de Janeiro, RJ, Brasil.

2. Médico Residente do $2^{\circ}$ ano de Cirurgia Plástica do Hospital dos Servidores do Estado do Rio de Janeiro, Rio de Janeiro, RJ, Brasil.

3. Médico Residente do $1^{\circ}$ ano de Cirurgia Plástica do Hospital dos Servidores do Estado do Rio de Janeiro, Rio de Janeiro, RJ, Brasil.

4. Membro Titular da Sociedade Brasileira de Cirurgia Plástica; Médico Staff do Hospital dos Servidores do Estado do Rio de Janeiro, Rio de Janeiro, RJ, Brasil.

5. Membro Titular da Sociedade Brasileira de Cirurgia Plástica; Chefe do Serviço de Cirurgia Plástica do Hospital dos Servidores do Estado do Rio de Janeiro, Rio de Janeiro, RJ, Brasil. 


\section{INTRODUÇÃO}

A gluteoplastia de aumento tem se tornado uma cirurgia mais requisitada pelos pacientes nos últimos anos no Brasil. Dados da Sociedade Brasileira de Cirurgia Plástica estimam que, de 2008 aos dias atuais, houve um aumento de $20 \%$ na realização do procedimento.

$\mathrm{O}$ uso de implantes para aumentar a região glútea trouxe interessantes opções para tratamento de hipotrofias moderadas e acentuadas dessa região ${ }^{1-4}$. Técnicas atuais, com a obtenção de melhores resultados e diminuição considerável das complicações, tornaram essa conduta cirúrgica opção satisfatória para melhorar o contorno corporal ${ }^{5-8}$. O aprimoramento técnico dessas operações trouxe novos horizontes e, com isso, a associação de lipoaspiração e enxertia associada tornou-se segura ${ }^{9-12}$.

Considerando-se a importância e crescimento dessa cirurgia em nossa especialidade ao longo dos últimos anos ${ }^{13}$, realizamos um trabalho cujo objetivo é analisar, prospectivamente, o ensino e o tratamento com gluteoplastia de aumento executado por residentes de um serviço público, supervisionados por seus preceptores, seus resultados e suas complicações associadas ao método.

\section{MÉTODO}

Estudo prospectivo de 20 pacientes tratados por residentes de Cirurgia Plástica, submetidos à gluteoplastia de aumento com prótese de silicone, no período de maio a outubro de 2010.

A técnica XYZ idealizada por Gonzalez ${ }^{14}$ contribuiu no sentido de estabelecer parâmetros anatômicos seguros para guiar o plano ideal de descolamento no interior do músculo glúteo máximo.

O procedimento foi iniciado com incisão na pele desde o ponto mais elevado do sulco interglúteo, de $7 \mathrm{~cm}$, retilínea, deixando ilha dérmica acima do ligamento sacrocutâneo em 5 casos. Após, seguiu-se a $45^{\circ}$, com descolamento subcutâneo em forma de coração invertido, de $7 \mathrm{~cm}$ de extensão, lateral, tendo como centro a incisão interglútea. Foram realizadas, a 2 $\mathrm{cm}$ da inserção sacral, incisão e divulsão romba com tesoura curva no sentido das fibras do músculo glúteo máximo, em 6 $\mathrm{cm}$ de comprimento, e 3 a $5 \mathrm{~cm}$ de profundidade, para permitir a passagem do descolador. Foi confeccionada uma loja com descolador rombo apropriado, no plano intramuscular, exatamente do tamanho do implante, como projeção da demarcação prévia na pele, conforme preconizado por Gonzales ${ }^{14}$. Previamente à retirada do descolador, foram introduzidos afastadores. Compressas com solução de adrenalina foram colocadas, realizando-se o mesmo procedimento do lado contralateral e, em seguida, retornou-se ao lado inicial, para revisão de hemostasia sem auxílio de fibra óptica. Em seguida, o implante foi introduzido e acomodado.
Foram utilizados 13 pares de implantes no formato redondo e 7 pares ovais. A rafia do músculo foi feita com fio Vicryl 3.0 em pontos simples, englobando ambas as margens musculares, ficando os fios reparados, e os nós, realizados somente após a inclusão do implante. $\mathrm{O}$ subcutâneo da área descolada foi suturado com pontos de adesão, fixados à fáscia profunda com Vicryl 3.0. A síntese de pele foi feita com pontos separados subdérmicos de ambos os lados da incisão interglútea, ancorados abaixo da ilha dérmica no ligamento sacrocutâneo, com intuito de recriar a prega interglútea e diminuir a tensão na incisão. A pele foi fechada com Mononylon 4.0 intradérmico.

A lipoaspiração, visando à harmonização das áreas vizinhas com a região aumentada, foi associada ou não, de acordo com o julgamento quanto à sua necessidade, sendo prioritariamente realizada na região sacral, flancos e culotes. A gordura lipoaspirada foi usada para lipoenxertia de depressão trocanteriana nos casos merecedores.

Não foram usados drenos. Foi realizada antibioticoprofilaxia com 2 gramas de Kefazol pré-operatoriamente e Cefalexina por 7 dias no pós-operatório. Os pacientes foram orientados a deambular precocemente, dormir em decúbito lateral ou ventral por 10 dias. Os cuidados com a ferida operatória foram: banho com degermante após miç̧ão ou evacuação e curativo fazendo limpeza com álcool $70 \%$ e fita microporosa unindo uma nádega à outra. Os pacientes no momento estão com 8 meses de acompanhamento.

\section{RESULTADOS}

Dentre os 20 pacientes avaliados, 19 eram do sexo feminino e 1 do sexo masculino, com média de idade foi 33,9 anos, variando de 22 a 60 anos.

A média do volume do implante foi $307 \mathrm{ml}$, com variação de 270 a $350 \mathrm{ml}$, sendo 7 ovais e 13 redondos. Praticou-se a lipoaspiração associada em seis (30\%) pacientes e lipoenxertia em quatro $(20 \%)$. A correção de lipodistrofia com prótese em pacientes com HIV foi realizada em 1 paciente, fato ocorrido pelas alterações do metabolismo lipídico dos medicamentos antirretrovirais ${ }^{15}$.

As complicações observadas foram quatro seromas, seguidos de três deiscências de pele parcial e uma deiscência total, tratados com curativo local de Kollagenase e fechamento por segunda intenção. Houve um caso de hematoma coagulado no oitavo dia de pós-operatório, sendo feita reabordagem, com limpeza, hemostasia e recolocação do implante no mesmo ato, com sucesso pós-operatório.

As Figuras 1 a 10 ilustram o tratamento realizado em alguns pacientes. 


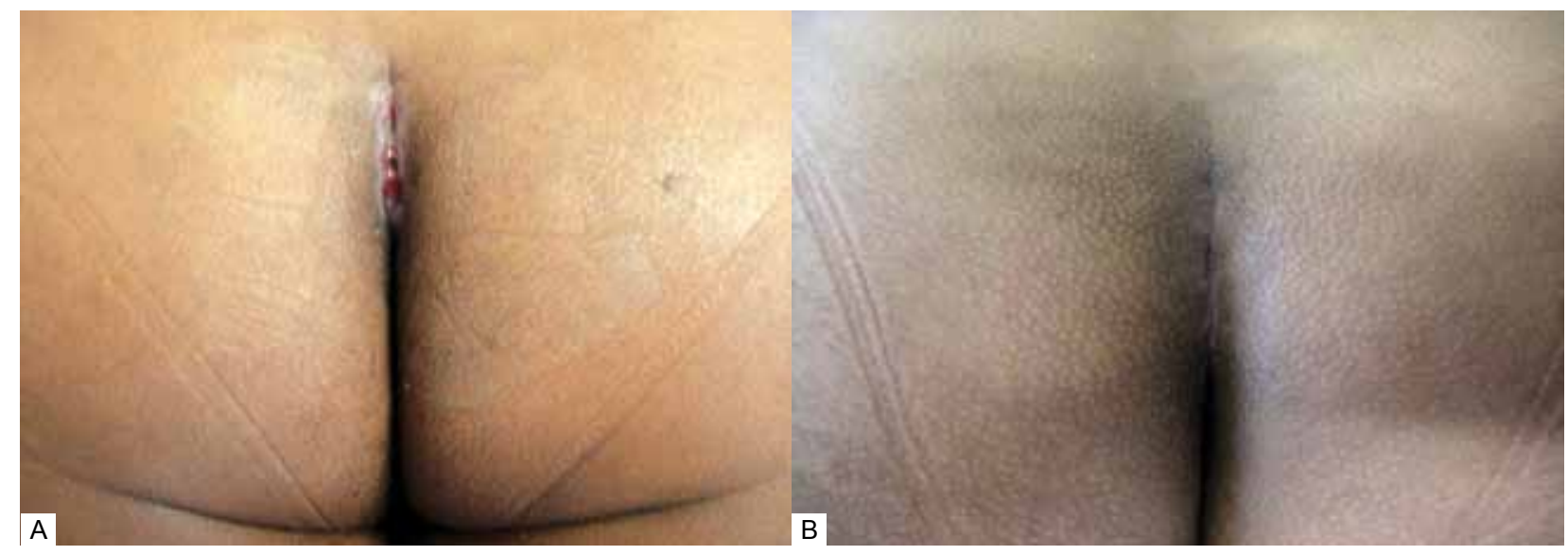

Figura 1 -A: Deiscência total de pele tratada com curativos locais. B: Aspecto final da cicatriz.

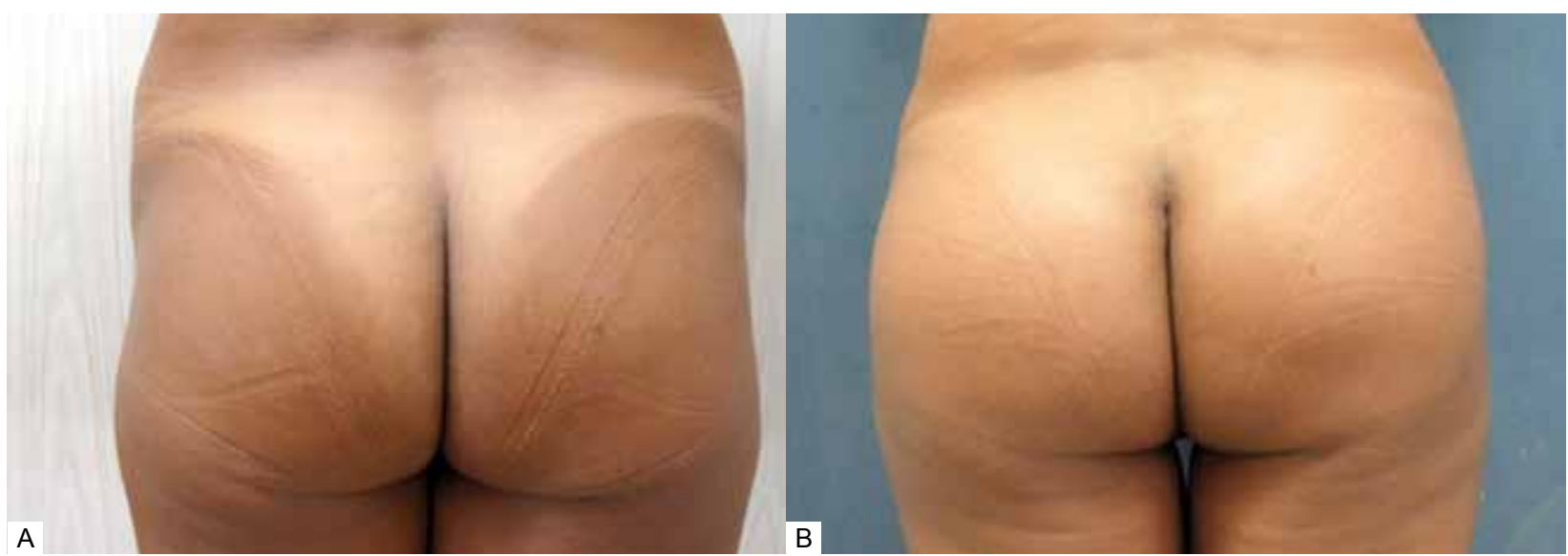

Figura 2 - A: Depressão trocantérica acentuada e lipodistrofia de flancos. B: Pós-operatório de 3 meses: implante glúteo $300 c c$ redondo e lipoenxertia trocantérica.

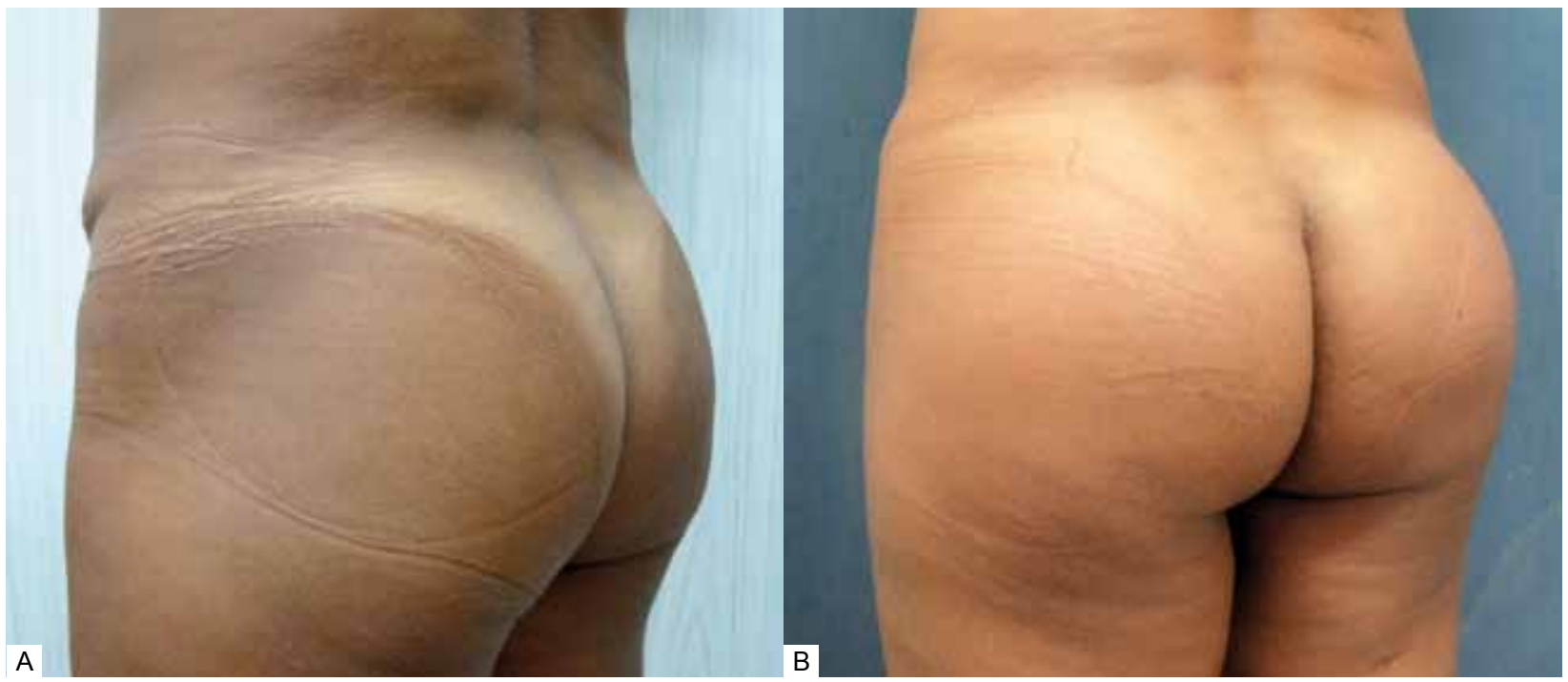

Figura 3 - A: Aspecto pré-operatório, vista obliqua posterior esquerda. B: Pós-operatório de 3 meses. 


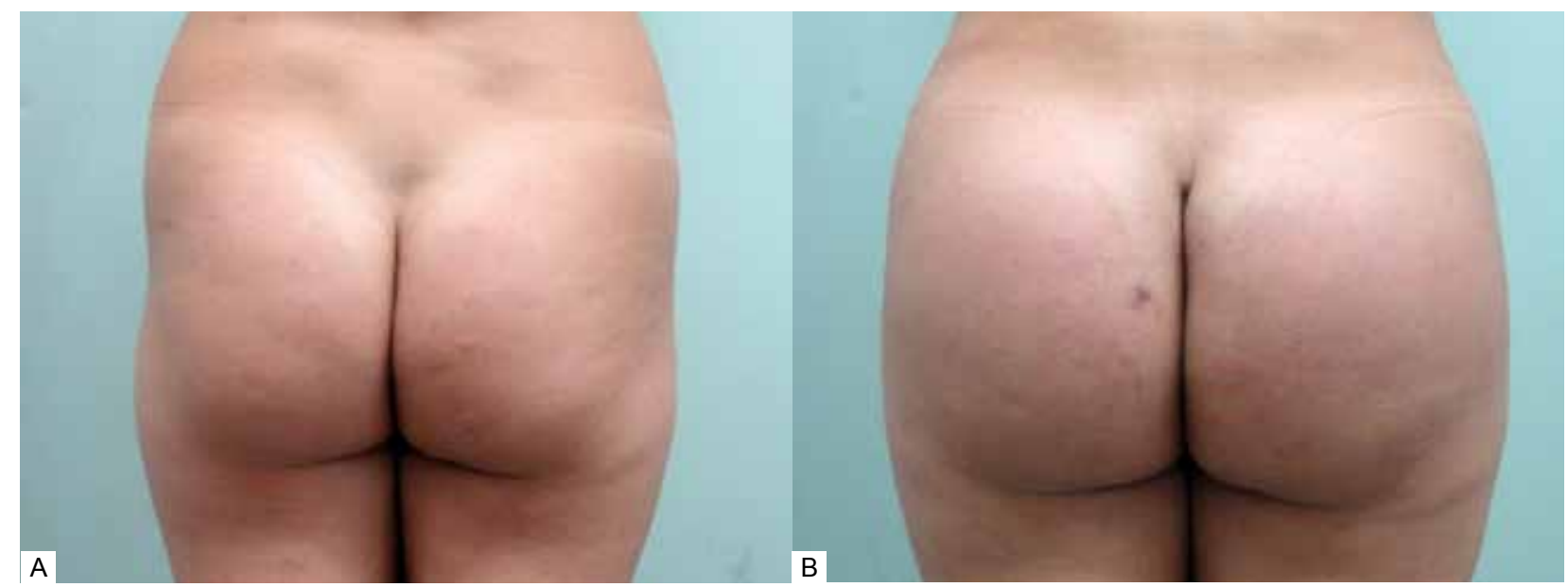

Figura 4-A: Hipotrofia glútea moderada com lipodistrofia de flancos e depressão trocanteriana. B: Pós-operatório de 4 meses: implante glúteo oval 300 cc e lipoaspiração de flancos com lipoenxertia da depressão trocantérica.

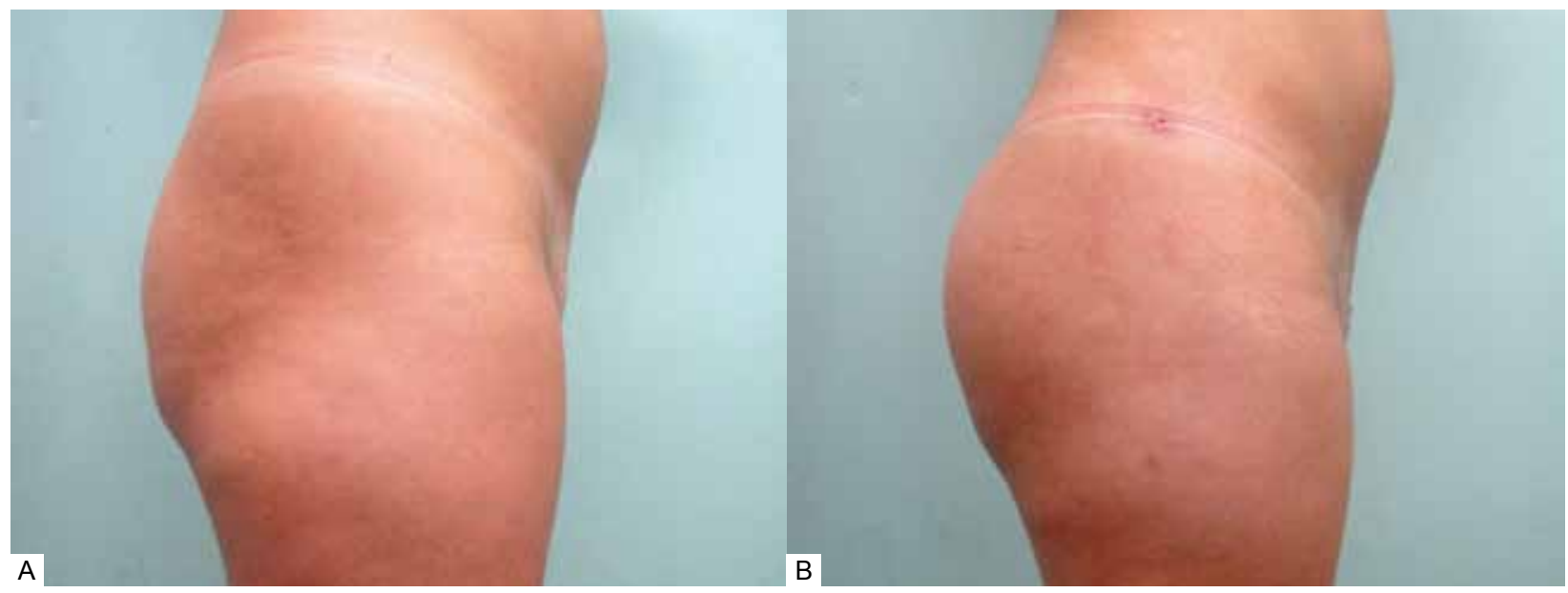

Figura 5-A: Pré-operatório, vista perfil. B: Pós-operatório, vista perfil.

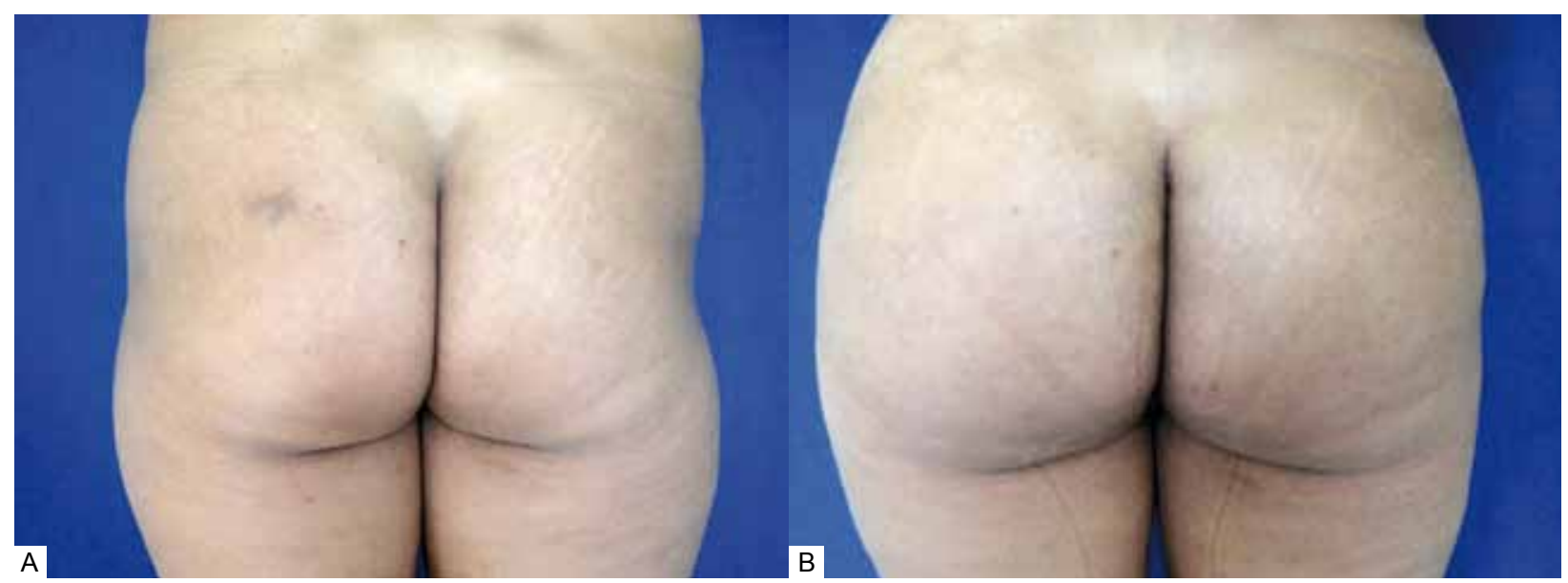

Figura 6-A: Acentuada depressão trocanteriana e lipodistrofia de culote e flancos. B: Aspecto pós-operatório 60 dias pós lipoaspiração e lipoenxertia associadas a implante de prótese glútea oval de 350cc posicionada obliquamente. 


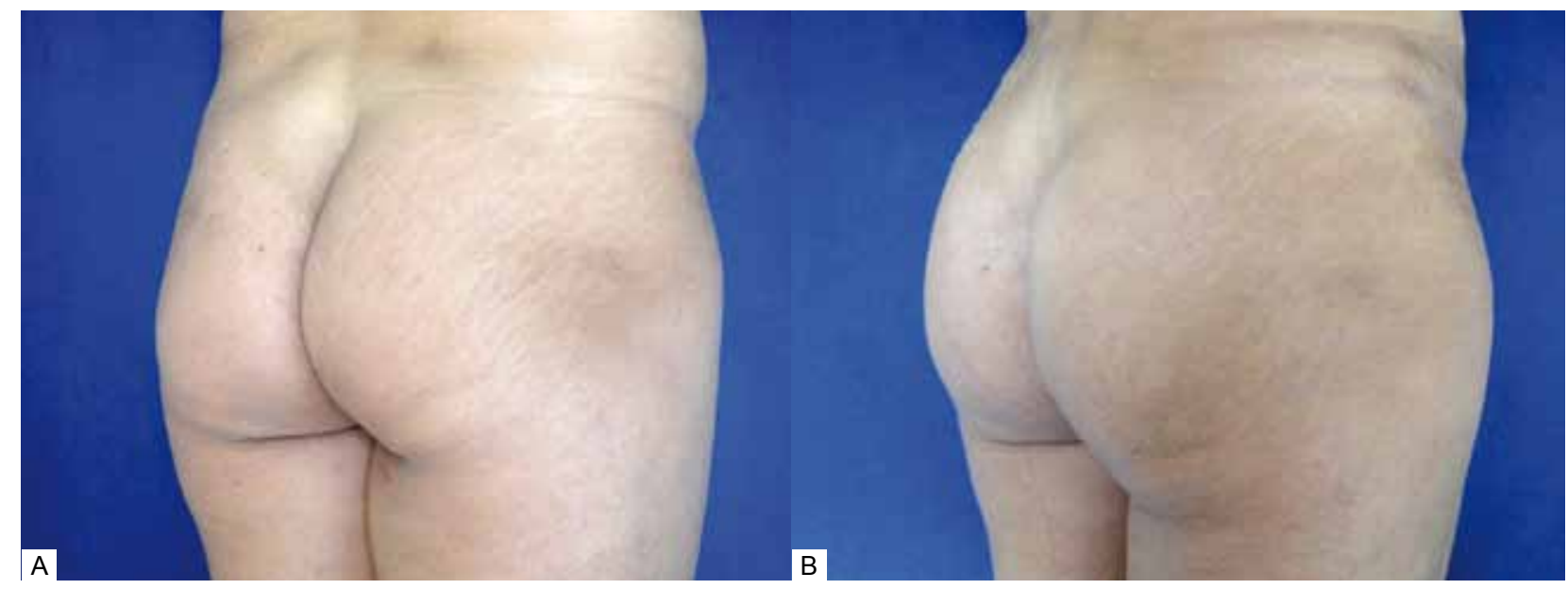

Figura 7-A: Pré-operatório oblíquo posterior direito. B: Pós-operatório de 3 meses.

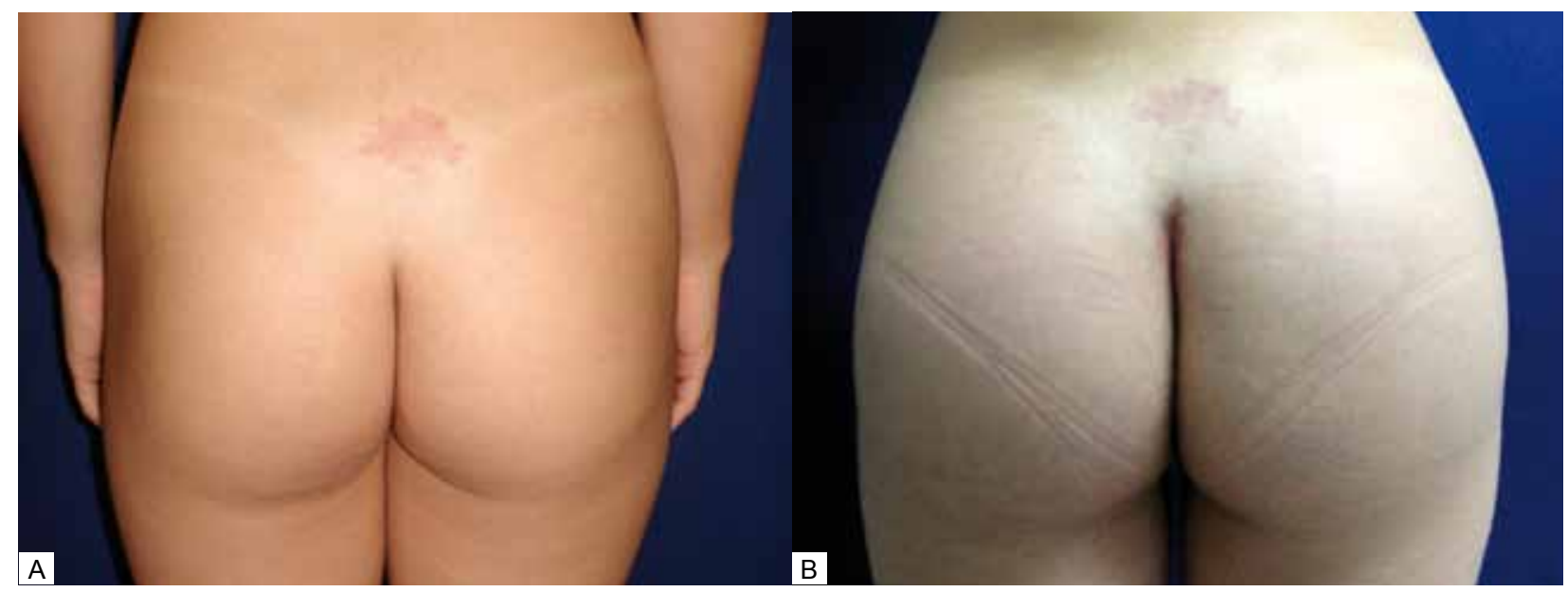

Figura 8 - A: Discreta depressão trocanteriana com moderada hipotrofia glútea. B: Prótese 350 cc oval disposta em $45^{\circ}$ com a linha médio-corporal obliquamente borda menos para baixo, a fim de preencher a leve depressão trocanteriana.

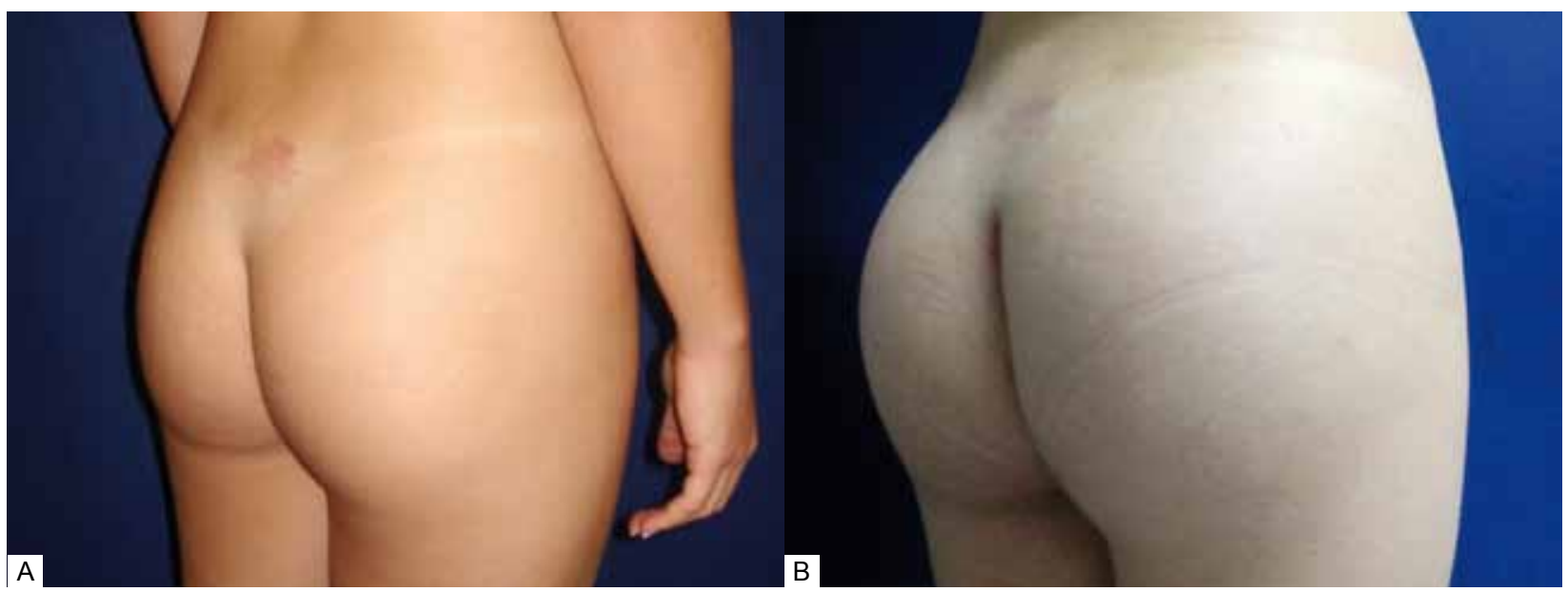

Figura 9-A: Pré-operatório: hipotrofia moderada de glúteo. B: Pós-operatório de 3 meses com resultado satisfatório somente com implante glúteo. 


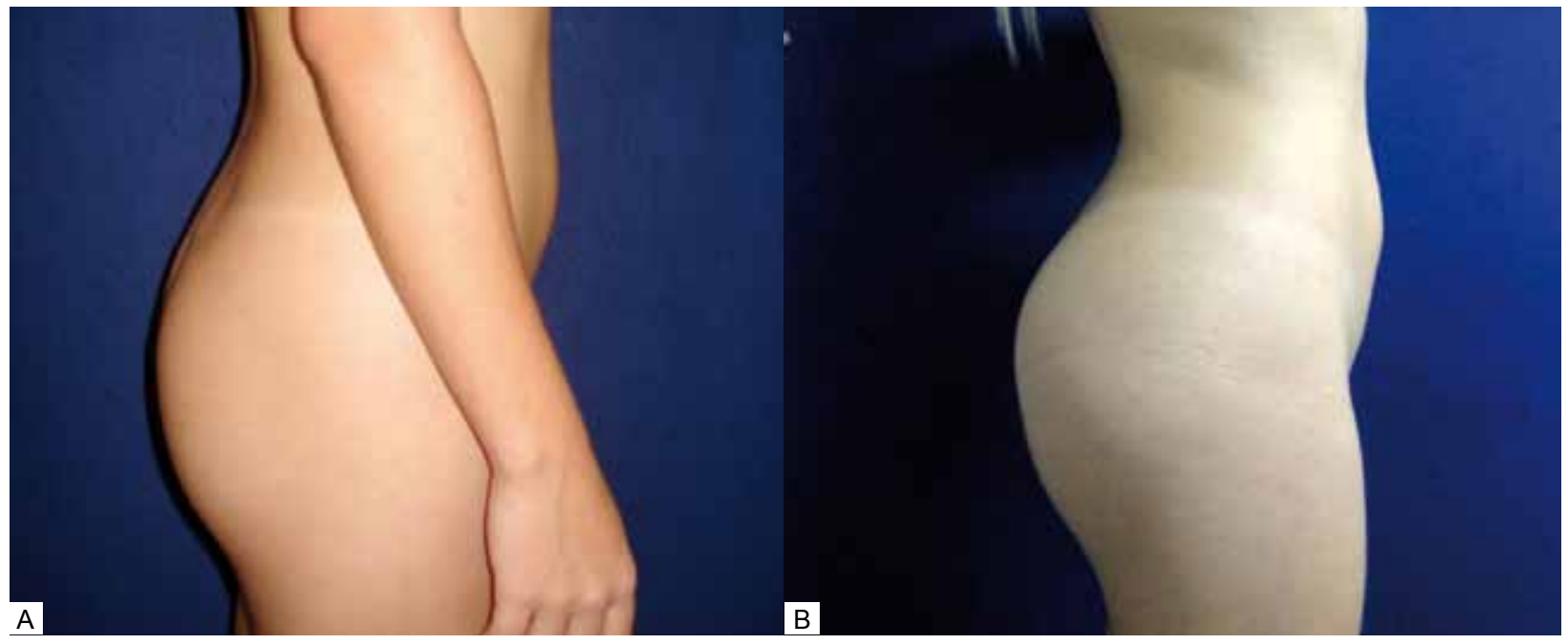

Figura 10 - A: Pré-operatório, vista de perfil. B: Pós-operatório, vista de perfil.

\section{DISCUSSÃO}

Observamos que o receio do descolamento às cegas do plano intramuscular, por vezes, pode inibir o começo do aprendizado em gluteoplastia. Entretanto, guiados por parâmetros técnicos e anatômicos precisos e sob supervisão qualificada, conseguiu-se a realização da cirurgia no plano intramuscular adequado em todos os casos, o que proporcionou ótima cobertura do implante e resultados naturais.

A associação de lipoaspiração ao implante glúteo em 30\% dos casos possibilitou também a correção de lipodistrofias sacrais, de flancos e culotes, na busca de uma relação mais harmônica da cintura com o quadril, melhorando o contorno corporal. Em 3 casos, nos quais a depressão trocanteriana era acentuada, optamos pela colocação do implante oval, oblíquo, com inclinação de $45^{\circ}$ à linha médio corporal, sendo sua porção maior na parte superior e a menor preenchendo a depressão lateral. Ainda nesses 3 casos, associamos a lipoenxertia na depressão após a inclusão da prótese, e obtivemos notável melhora na relação do contorno trocanteriano com a nova projeção glútea estabelecida pelo implante.

A busca por melhores resultados trouxe à tona a possibilidade de associação dessas técnicas, já que a correção das lipodistrofias não passa apenas pela retirada de tecido adiposo, mas também pela inclusão de volumes em certas regiões. Essa associação técnica tem poucos relatos na literatura, optando os cirurgiões, na maioria dos casos, por executar apenas um dos procedimentos. O correto diagnóstico e conhecimento técnico levarão ao tratamento adequado das deformidades presentes $^{12}$

O caso de hematoma coagulado no oitavo pós-operatório, muito provavelmente, deveu-se ao não respeito ao repouso prescrito pelo médico assistente, o que foi corroborado pelas informações da paciente. Entretanto, o caso teve evolução satisfatória.

Conforme afirma Gonzales ${ }^{7}$, a deiscência em seguida ao seroma é mais comum que a deiscência isolada. Os três casos de deiscência que apresentamos ocorreram após seroma; é interessante observar que em todos esses pacientes foi deixada a ilha dérmica. Ressaltamos a necessidade de maior cuidado na confecção desse pedículo dérmico, para que o mesmo não sofra isquemia e isole ambos os lados de colocação das próteses, bem como evitar-se tensão em sua sutura, aspectos que devem ser observados com rigor quando se pretende deixar a ilha dérmica.

Também vale ressaltar que, ao deixarmos os pontos de reparo passados em ambas as bordas musculares com Vicryl 3.0, antes de colocarmos o implante, sentimos maior facilidade e segurança no momento de fechamento da loja muscular com o implante já posicionado, o que diminui o risco de perfuração do implante na passagem do ponto.

Alcançamos a satisfação de todos os pacientes, mesmo nos casos com complicações, o que demonstra que o cuidado na execução da técnica e seguimento pós-operatório foram realizados de forma adequada pelos residentes.

Queixas de dor estiveram presentes na maioria dos casos, principalmente na primeira semana de pós-operatório, o que foi se tornando diminuta com o passar do tempo da cirurgia e acomodação do implante.

\section{CONCLUSÃO}

Os resultados obtidos demonstram a grande aplicabilidade do ensino da gluteoplastia, bem como de sua associação segura com a lipoaspiração de áreas satélites ao glúteo e lipoenxertia cautelosa da depressão trocanteriana em casos 
selecionados, o que valoriza o resultado estético da prótese glútea e evidencia ótimos resultados no contorno corporal. Os índices de complicação e a resolução das mesmas são aceitáveis, comparáveis aos da literatura.

Sabemos que a referida cirurgia ainda não é realizada e ensinada na prática, com frequência, em muitos serviços de residência em Cirurgia Plástica do Brasil. Assim, cabe aqui salientar a importância de se aumentar e estimular o aprendizado prático da gluteoplastia com implantes aos futuros médicos residentes, considerando-se o aumento observado na requisição recente e segurança de execução da mesma, quando respeitados os princípios técnicos atuais. Sem dúvida, esse treinamento sistemático engrandecerá ainda mais a nossa especialidade.

\section{REFERÊNCIAS}

1. Souza Pinto EB. Novos conceitos em lipoenxertia. Lipoaspiração superficial. Rio de Janeiro:Revinter;1999. p.97-101.

2. Gonzalez R. Prótese para a região glútea. In: Tournieux A, ed. Atualização em cirurgia plástica. São Paulo: Robe Editorial;1994. p.555-70.

3. Pereira LH, Radwanski HN. Fat grafting of the buttocks and lower limbs. Aesthetic Plast Surg. 1996;20(5):409-16.
4. Perén PA, Gómez JB, Guerrerosantos J, Salazar CA. Gluteus augmentation with fat grafting. Aesthetic Plast Surg. 2000;24(6):412-7.

5. Robles JM, Tagliapietra JC, Grandi M. Gluteoplastia de aumento: implante submuscular. Cir Plast Iberolat. 1984;10(4):365-9.

6. Vergara R, Marcos M. Intramuscular gluteal implants. Aesthetic Plast Surg. 1996;20(3):259-62.

7. Gonzalez R. Augmentation gluteoplasty: the XYZ method. Aesthetic Plast Surg. 2004;28(6):417-25.

8. MartinsLC.Plasticsurgery: foundations andart. SãoPaulo:MEDSI;2003. p.755-65.

9. Cardenas Restrepo JC, Muñoz Ahmed JA. Large-volume lipoinjection for gluteal augmentation. Aesthetic Surg J. 2002;22(1):33-8.

10. Murillo WL. Buttock augmentation: case studies of fat injection monitored by magnetic resonance imaging. Plast Reconstr Surg. 2004;114(6):1606-14.

11. Roberts TL 3rd, Toledo LS, Badin AZ. Augmentation of the buttocks by micro fat grafting. Aesthetic Surg J. 2001;21(4):311-9.

12. Cárdenas-Camarena L, Paillet JC. Combined gluteoplasty: liposuction and gluteal implants. Plast Reconstr Surg. 2007;119(3):106774.

13. de la Peña JA, Rubio OV, Cano JP, Cedillo MC, Garcés MT. History of gluteal augmentation. Clin Plast Surg. 2006;33(3):307-19.

14. Gonzalez R. Buttocks reshaping: posterior contour surgery. A step-bystep approach. Rio de Janeiro:Indexa;2006.

15. Moreno S, Miralles C, Negredo E, Domingo P, Estrada V, Gutiérrez F, et al. Disorders of body fat distribution in HIV-1-infected patients. AIDS Rev. 2009;11(3):126-34.

\section{Correspondência para:}

